\title{
Ultrahigh-density spin-polarized hydrogen isotopes from the photodissociation of hydrogen halides: new applications for laser-ion acceleration, magnetometry, and polarized nuclear fusion
}

\author{
Alexandros K. Spiliotis $\mathbb{B}^{1,2}$, Michalis Xygkis ${ }^{1,2}$, Michail E. Koutrakis ${ }^{1,2}$, Konstantinos Tazes ${ }^{1,2}$, Gregoris K. Boulogiannis ${ }^{1,2}$,

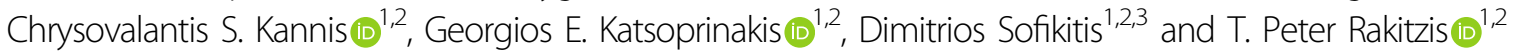

\begin{abstract}
Recently, our group produced spin-polarized hydrogen (SPH) atoms at densities of at least $10^{19} \mathrm{~cm}^{-3}$ from the photodissociation of hydrogen halide molecules with circularly polarized UV light and measured them via magnetization-quantum beats with a pickup coil. These densities are approximately 7 orders of magnitude higher than those produced using conventional methods, opening up new fields of application, such as ultrafast magnetometry, the production of polarized MeV and GeV particle beams, such as electron beams with intensities approximately $10^{4}$ higher than current sources, and the study of polarized nuclear fusion, for which the reaction cross sections of D-T and $\mathrm{D}_{-}{ }^{3} \mathrm{He}$ reactions are expected to increase by $50 \%$ for fully polarized nuclear spins. We review the production, detection, depolarization mechanisms, and potential applications of high-density SPH.
\end{abstract}

\section{Introduction}

The production and manipulation of spin-polarized electrons and nuclei are fundamental to many fields of physics, including nuclear magnetic resonance (NMR), electron-spin resonance (ESR), magnetometry, spintronics, Bose-Einstein condensation (BEC), and in studying scattering and reactions in atomic, molecular, optical, particle, and nuclear physics ${ }^{1-7}$. However, the equilibrium polarization of a sample, for all but cryogenic temperatures, is very small (typically less than $10^{-5}$, even for large magnetic fields of approximately $\sim 1 \mathrm{~T}$ ). Therefore, the key to producing large polarizations in environments with rapid depolarization mechanisms is to polarize rapidly in situ (if possible) or to polarize in

\footnotetext{
Correspondence: T. Peter Rakitzis (ptr@iesl.forth.gr)

${ }^{1}$ Foundation for Research and Technology Hellas, Institute of Electronic

Structure and Laser, N. Plastira 100, Heraklion, Crete GR-71110, Greece

${ }^{2}$ University of Crete, Department of Physics, Herakleio, Greece

Full list of author information is available at the end of the article
}

conditions where depolarization is small, then to quickly introduce the polarized matter into the desired environment where the depolarization rates are high and to perform the desired experiments rapidly, before significant depolarization occurs. One example of this is the introduction of spin-polarized ${ }^{129} \mathrm{Xe}$ into the human body to enhance magnetic resonance imaging (MRI) signals for diagnostic purposes ${ }^{8}$. Thus, the time for the production of spin polarization, particularly in situ, is of paramount importance for applications of polarized matter.

Recently, our group produced spin-polarized hydrogen $(\mathrm{SPH})$ and deuterium (SPD) atoms at densities of at least $10^{19} \mathrm{~cm}^{-3}$ and on the $100 \mathrm{ps}$ timescale from the photodissociation of hydrogen halides 9 . These densities and timescales are at least 7 orders of magnitude higher and faster, respectively, compared to conventional production methods of SPH. These new regimes of SPH density and near-instantaneous in situ polarization production open up a range of new applications of SPH. The aim of this 
review is to describe the production, detection, and depolarization of SPH in this ultrahigh-density regime and to describe the new applications that are now possible. Specifically, in section II, we provide the details of the photodissociation of hydrogen halides and explain why the SPH density can surpass conventional SPH production techniques. In section III, we describe the detection of SPH via the measurement of magnetization-quantum beats with a pickup coil. In section IV, we describe the depolarization mechanisms that limit the SPH lifetime, both for low-density $\mathrm{SPH}$ and for high-density $\mathrm{SPH}$. Finally, the last three sections deal with new applications: section $\mathrm{V}$ describes fast magnetometry, with ns time resolution; section VI describes the production of spinpolarized electron and proton beams from laser-plasma acceleration, with the potential to surpass the flux of current sources by 4 orders of magnitude ${ }^{10}$; and section VII describes proposals for testing polarized fusion, for the $\mathrm{D}-\mathrm{T}$ and $\mathrm{D}-{ }^{3} \mathrm{He}$ reactions, where it is expected that polarizing the nuclear spins will increase the fusion cross section by $50 \%$; until now, such tests have not been performed in a plasma due to the lack of sources that can produce SPD at sufficiently high density.

\section{SPH production}

The conventional methods for SPH production are the Stern-Gerlach spin-separation technique (or atomic beam source (ABS)) and spin-exchange optical pumping. We first describe these methods in some detail.

\section{Atomic beam source}

An ABS produces an atomic beam of unpolarized hydrogen atoms, which then passes through an inhomogeneous magnetic field, which induces a deflection force on the spin-up electrons and an opposite force on the spindown electrons. These forces allow the separation of the atoms with spin-up and spin-down electrons into separate beams. However, the time and distance needed for this beam separation are very long: the distance is on the order of $1 \mathrm{~m}$ for a few $\mathrm{mm}$ of beam separation, and the separation time is on the order of $1 \mathrm{~ms}$. The density of the beam must be kept below a critical limit, above which velocity-changing collisions between atoms diffuse the atomic beam to the point where spin separation is no longer spatially resolved. The critical density limit is approximately $10^{12} \mathrm{~cm}^{-3}$, and a typical beam velocity of $\sim 2000 \mathrm{~m} / \mathrm{s}$ and a beam crosssectional area of $\sim 0.1 \mathrm{~cm}^{2}$ yield an SPH production rate of nearly $10^{17} \mathrm{~s}^{-111}$. We note that these densities (less than $10^{-7}$ bar) and production rates (less than $1 \mu \mathrm{mol} \mathrm{s}^{-1}$ ) are microscopic and limit the range of application significantly.

\section{Spin-exchange optical pumping (SEOP)}

Optical pumping is a method for transferring polarization from photons to atoms for atoms with strong transitions that can be pumped with powerful lasers. The ground-state $\mathrm{H}$ atom's first transition is at $121.6 \mathrm{~nm}$ in vacuum UV light, which is not convenient for optical pumping (strong lasers do not exist there, among many other problems). The alkali atoms have strong transitions in the near IR and can be excited with circularly polarized $\sigma^{+}$radiation, which increases the angular momentum projection of the excited-state atom by one unit of $\hbar$. The atom then fluoresces, and some fraction of this polarization is retained, on average, in the ground state. This polarization cycle (optical excitation and fluorescence) needs to be repeated many times (approximately 10) until the atomic polarization (nuclear and electronic spin) approaches $100 \%$, and when performed with cw lasers, the polarization of the alkali atoms can be maintained continuously at a density of approximately $10^{13} \mathrm{~cm}^{-3}$. While these high-density polarized alkali atoms do not have any direct application (beyond fundamental research), they can be used as a polarization source to transfer polarization to other atoms through collisions ${ }^{12,13}$, termed SEOP. SEOP has been used to produce SPH at production rates of approximately $10^{17} \mathrm{~s}^{-1}$, similar to ABS. The main application of SEOP is the production of spin-polarized noble gases with nuclear spins (mainly ${ }^{3} \mathrm{He}$ and ${ }^{129} \mathrm{Xe}$ ) in macroscopic quantities $(\sim 10 \mathrm{~L} \mathrm{bar} / \mathrm{h})$, which are used for MRI signal enhancement. The success with ${ }^{3} \mathrm{He}$ and ${ }^{129} \mathrm{Xe}$ is mainly due to the very slow depolarization rates of these noble gases, as the nuclei are protected from depolarization due to the closed-shell electronic structure. However, similar to $\mathrm{ABS}$, the polarization time for SEOP is very slow and cannot polarize open-shell atoms at high densities.

\section{SPH from photodissociation}

The production of spin-polarized atoms from diatomic molecule photodissociation has significant similarities to both ABS and SEOP but also important differences. We first provide details on the photodissociation mechanism before drawing these comparisons.

Spin-polarized atoms can be produced from the photodissociation of diatomic molecules with circularly polarized light ${ }^{14-16}$ because the electronic angular momentum projection of a particular molecular state adiabatically correlates to particular $\mathrm{m}$ states of the separated atoms ${ }^{17,18}$. In particular, the total electronic angular momentum projection quantum number $\Omega$ is equal to the sum of the $\mathrm{m}$ states of the separated atoms $\mathrm{m}_{\mathrm{A}}$ and $\mathrm{m}_{B}$ :

$$
\Omega=m_{A}+m_{B}
$$

Hydrogen halides, HY, have been shown to be the most effective molecules for the production of $\mathrm{SPH}^{19-29}$, as they are the only diatomic molecules that contain $\mathrm{H}$ 


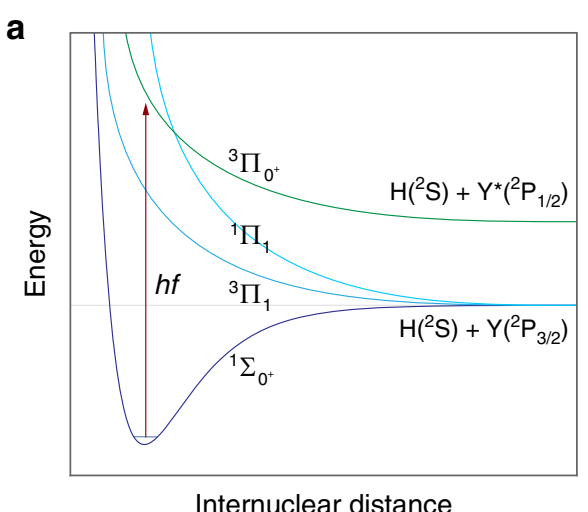

b

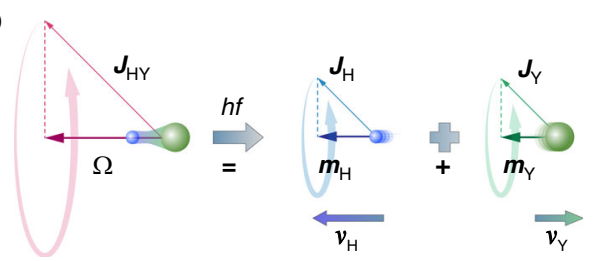

Fig. 1 Correlation of molecular electronic states to atomic $\mathrm{m}$ states. a The potential energy curves that participate in the photodissociation HY. b The total electronic angular momentum projection $(\Omega)$ of $\mathrm{HY}$ is conserved during the dissociation and is equal to the sum of the product $m$ states: $\Omega=m_{A}+m_{B}$

atoms except for $\mathrm{H}_{2}$ (which photodissociates below $100 \mathrm{~nm}$, where high photon fluxes cannot be produced). The potential energy curves of the three lowest electronic states of HY that play a role in the photodissociation and photofragment polarization are shown in Fig. $1, A^{1} \Pi_{1}, a$ ${ }^{3} \Pi_{1}, a{ }^{3} \Pi_{0+}$, as well as the ground state $\mathrm{X}^{1} \Sigma_{0+}{ }^{30-33}$. These three states correlate adiabatically to the atomic $\mathrm{m}$ states as

$$
\begin{aligned}
\mathrm{HY}\left(A^{1} \prod_{1} ; \Omega= \pm 1\right) \rightarrow & \mathrm{H}\left(\mathrm{m}_{\mathrm{H}}=\mp 1 / 2\right) \\
& +\mathrm{Y}\left(\mathrm{m}_{\mathrm{Y}}= \pm 3 / 2\right) \\
\mathrm{HY}\left(a^{3} \prod_{1} ; \Omega= \pm 1\right) \rightarrow & \mathrm{H}\left(\mathrm{m}_{\mathrm{H}}= \pm 1 / 2\right) \\
& +\mathrm{Y}\left(\mathrm{m}_{\mathrm{Y}}= \pm 1 / 2\right) \\
\mathrm{HY}\left(a^{3} \prod_{0+} ; \Omega=0\right) \rightarrow & \mathrm{H}\left(\mathrm{m}_{\mathrm{H}}= \pm 1 / 2\right) \\
& +Y^{*}\left(\mathrm{~m}_{\mathrm{Y}^{*}}=\mp 1 / 2\right)
\end{aligned}
$$

where $\mathrm{Y}$ refers to ground-state $\mathrm{Y}\left({ }^{2} \mathrm{P}_{3 / 2}\right)$ atoms and $\mathrm{Y}^{*}$ refers to spin-orbit-excited $\mathrm{Y}\left({ }^{2} \mathrm{P}_{1 / 2}\right)$ atoms.

For maximum polarization and for ease of understanding, the molecular bonds can be aligned along the polarization direction of the photodissociation laser using the method of strong-field laser alignment. For such bond-aligned molecules and for a circularly polarized $\sigma^{+}$ photodissociation laser, the allowed transitions are from the ground state $\Omega=0$ to the $\Omega=+1 A{ }^{1} \Pi_{1}$ and $a^{3} \Pi_{1}$ states, as well as to the $\Omega=0 a^{3} \Pi_{0+}$ state. We observe, from Eqs. (2-4), that, for adiabatic dissociation, the $A^{1} \Pi_{1}$ state yields $\mathrm{H}$ atoms with electrons that are spin down, the $a^{3} \Pi_{1}$ state yields $\mathrm{H}$ atoms with electrons that are spin up, whereas the $a^{3} \Pi_{0+}$ state yields $\mathrm{H}$ atoms with electrons that are unpolarized. Therefore, one can produce $\mathrm{H}$ atoms with $100 \%$ spin-polarized electrons, from excitation to the $A^{1} \Pi_{1}$ or $a^{3} \Pi_{1}$ states, followed by adiabatic dissociation (but not from exciting a superposition of the $A^{1} \Pi_{1}$ and $a$ ${ }^{3} \Pi_{1}$ states). Excitation of the ${ }^{3} \Pi_{0+}$ state (which yields unpolarized $\mathrm{H}$ atoms) is avoided if the molecular bonds are aligned parallel to the propagation direction of the circularly polarized photodissociation laser.

For all hydrogen halide (HY) molecules, it is possible to excite, almost exclusively, the $A^{1} \Pi_{1}$ state, or the $a^{3} \Pi_{1}$ state, with a large absorption cross section in the UV. For example, $\mathrm{HI}$ can be photodissociated almost exclusively through the $A^{1} \Pi_{1}$ state at $213 \mathrm{~nm}$ and through the $a^{3} \Pi_{1}$ state at $266 \mathrm{~nm}$. Therefore, conditions exist where the excitation step is fully compatible with the production (near) of $100 \%$ spin-polarized $\mathrm{H}$ atoms.

Nonadiabatic transitions during dissociation can also occur, which is an additional potential complication. These nonadiabatic transitions transfer the population from one state to another at long interatomic distances, and they involve spin flips in both atomic photofragments while maintaining the condition of Eq. (1). For example, after exclusive excitation to the $A^{1} \Pi_{1}$ state (which adiabatically yields SPH with spin-down electrons), nonadiabatic transfer to the $a^{3} \Pi_{1}$ state yields SPH with spin$u p$ electrons. Clearly, a large mixture of adiabatic and nonadiabatic dissociation strongly reduces the spin polarization, and either (near-)complete adiabatic or nonadiabatic dissociation is desirable to produce highly spin-polarized $\mathrm{H}$ atoms. It would be useful if theoretical calculations could accurately predict the contribution of nonadiabatic transitions; while successful in the case of $\mathrm{HCl}$, the contributions of nonadiabatic transitions were not treated properly for $\mathrm{DI}^{34}$. Fortunately, it has been shown experimentally that highly spin-polarized $\mathrm{H}$ or $\mathrm{D}$ atoms (with polarizations above $70 \%$ ) are produced from photodissociation at wavelengths where powerful UV lasers exist: from $\mathrm{HCl}$ and $\mathrm{HBr}$ at $193 \mathrm{~nm}$, from $\mathrm{HCl}, \mathrm{HBr}$, and DI at $213 \mathrm{~nm}$, and from DI at $266 \mathrm{~nm}$.

\section{Comparison of the photodissociation method to an ABS and SEOP}

The photodissociation method involves both optical pumping and polarization through strong gradients of fields, so it can be compared directly to an ABS and SEOP. Optical pumping is relatively inefficient with the use of photons, as approximately 10 are needed to polarize the pumped atom, and many more are needed to maintain the polarization for the duration of the experiment. In contrast, the photodissociation method is maximally efficient, as it 
can produce SPH with an electron polarization of $100 \%$ with only one photon. The ABS spin separates a beam by having large magnetic-field gradients over a few $\mathrm{cm}$ and then accumulates a beam separation over approximately $\sim 1 \mathrm{~m}$ in $\sim 1 \mathrm{~ms}$. The photodissociation method separates the energetic paths of the m-states of the atoms from the gigantic electric fields between the two nuclei over a distance of less than $1 \mathrm{~nm}$ and in $\sim 100 \mathrm{fs}$. The fact that the polarization time is approximately 9 orders of magnitude faster than that of the ABS suggests that the photodissociation method should operate at pressures approximately 9 orders of magnitude higher or at densities up to approximately $10^{21} \mathrm{~cm}^{-3}$; the observation of SPD in excess of $10^{19} \mathrm{~cm}^{-39}$ and recent measurements of $\mathrm{SPH}$ at $\mathrm{HCl}$ densities of at least $10^{20} \mathrm{~cm}^{-335}$ support this prediction. We describe the method for detecting these high SPH densities in the following section and the observed depolarization mechanisms in section IV.

\section{SPH detection}

\section{SPH optical detection}

SPH from photodissociation was first detected optically with two different methods. Before polarizationsensitive detection schemes for SPH became available, the SPH was measured indirectly: upon prompt photodissociation of $\mathrm{HCl}$ with circularly polarized light at $193 \mathrm{~nm}$, both $\mathrm{H}$ and $\mathrm{Cl}$ atom cofragments are polarized, and the degree of $\mathrm{H}$ polarization can be inferred from the $\mathrm{Cl}$ polarization using Eq. (1). The polarization of the $\mathrm{Cl}$ atoms was measured using $(2+1)$ resonanceenhanced multiphoton ionization (REMPI), using two photons to excite the $\mathrm{Cl}$ atoms to an intermediate state and one photon for ionization. The angular distribution of the $\mathrm{Cl}$ ion signal was measured with slice imaging, and the dependence of the angular distribution of the ions on the polarization directions of the photolysis and probe lasers provided sufficient information for the determination of the $\mathrm{Cl}$ polarization. The $\mathrm{H}$ atoms were inferred to have a polarization of approximately $72 \%{ }^{19}$. Shortly afterwards, one of the authors proposed various polarized fluorescence methods to detect SPH directly ${ }^{20}$. This was achieved on the $1 \mathrm{~s}-2 \mathrm{p}$ fluorescence transition at $121.6 \mathrm{~nm}^{23,24}$ and on the $2 \mathrm{p}-3 \mathrm{~d}$ transition (followed by the two-photon $1 \mathrm{~s}-3 \mathrm{~d}$ excitation) at $656 \mathrm{~nm}^{25}$. The polarization of the fluorescence experiments agrees with the indirect REMPI experiments and with calculations. However, the optical detection methods are limited to low pressures of approximately $0.01 \mathrm{mbar}$ (as the excited state is depolarized by collisions) and to low SPH densities of approximately $10^{12} \mathrm{~cm}^{-3}$ (at which point the optical transitions become optically thick, and light does not reach the detector). Therefore, it was not possible to optically measure SPH at high density to determine the upper limits possible with the photodissociation method. Crude estimates of the SPH depolarization cross sections indicated that SPH with a density of at least $10^{16} \mathrm{~cm}^{-3}$ may have a lifetime of $>10 \mathrm{~ns}^{22}$, but these could not be confirmed optically.

\section{SPH pickup-coil detection}

Samples with large time-dependent magnetization can be detected with a pickup coil, such as in NMR. However, the principle of detecting spins with a pickup coil, which has been produced with a short-pulsed laser, was demonstrated recently by Milner et al. ${ }^{36}$ from the production of spin-polarized $\mathrm{O}_{2}$ molecules from the centrifuging of $\mathrm{O}_{2}$ at $\sim 1$ bar pressure with a tailored fs pulse. This demonstration inspired the measurement of SPH with a pickup coil ${ }^{9}$.

The experimental setup is shown in Fig. 2: a pickup coil with $N \sim 4$ turns, length $L \sim 5 \mathrm{~mm}$ and diameter $\sim 2 \mathrm{~mm}$ is placed in a small vacuum chamber. A $150 \mathrm{ps}, 213 \mathrm{~nm}$ or $266 \mathrm{~nm}$, circularly polarized light pulse, with a pulse energy of 1-10 mJ, passes through the coil and photodissociates hydrogen halide gas at typical pressures of approximately 0.1-5 bar. For near optically-thick samples, up to approximately $10 \%$ of the light pulse can be absorbed in the coil, which corresponds to approximately $10^{13}-10^{15}$ photons, producing $10^{13}-10^{15} \mathrm{SPH}$ atoms. The electrons of the $\mathrm{H}$ atoms are initially predominantly spin up, whereas the protons are unpolarized. The electron polarization is transferred to the protons and back at the hyperfinebeating frequency so that the electron magnetization $M(t)$ is given by

$$
M(t)=M_{n} \mathrm{e}^{-t / \tau} \cos ^{2}\left(\frac{\omega t}{2}\right)
$$

where $\tau$ is the polarization lifetime, $\omega=\left(E_{1}-E_{0}\right) / \hbar$ is the angular hyperfine frequency, $E_{\mathrm{F}}$ are the energies of the hyperfine states $F=0$ and $1, M_{n}=g_{S} m_{S} n P \mu_{B} \approx n P \mu_{B}$ is the magnetic moment of $n$ spin-polarized electrons with initial polarization $P$ (where $-1 \leq P \leq 1$ ), $\mu_{B}$ is the Bohr

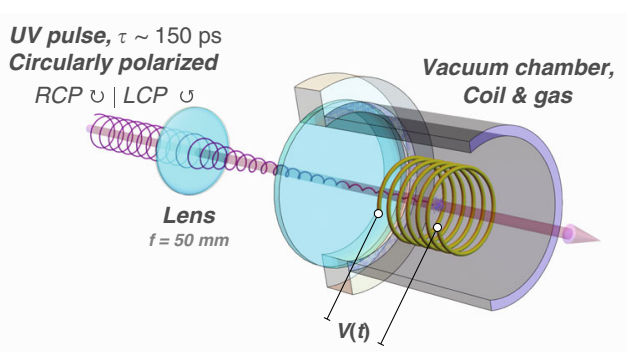

Fig. 2 Experimental setup for the detection of SPH with a pickup coil. A circularly polarized, 150 ps UV pulse (at 213 or $266 \mathrm{~nm}$ ) is focused into a vacuum chamber containing hydrogen halide gas at $0.1-5$ bar, and passes through the pickup coil, and produces the signal $V(t)$ 
magneton, $g_{S} \approx 2$ is the electron $g$ factor, and $m_{s}=1 / 2$ is the electron-spin projection. The exact form of $M(t)$ is given by convoluting Eq. (5) with the pulse width of the photodissociation laser, as shown in Fig. 3.

The time-dependent magnetic flux created through the coil causes a time-dependent voltage in the coil $V(t)$. In simulating the detection apparatus, $V(t)$ is treated as a time-dependent voltage source connected in series with a coil of inductance $L$ (and with a negligible resistance) and a load resistor (the $50 \Omega$ resistor of the $3 \mathrm{GHz}$ oscilloscope (Rohde \& Schwarz RTO2034) used in the experiment. The $V(t)$ signal created in the coil, including that resulting from Lenz's law, is the solution of the differential equation:

$$
V(t)=\mu_{0} \frac{N}{l} \frac{\mathrm{d} M(t)}{\mathrm{d} t}-\frac{L}{R} \frac{\mathrm{d} V(t)}{\mathrm{d} t}
$$

where $l$ is the coil length and $N$ is the number of coil turns. In Fig. 4a, an experimental magnetization-quantum beat signal is shown from SPH produced from the photodissociation of $\mathrm{HBr}$ at $75 \mathrm{mbar}$, with an exponential-decay lifetime $\tau_{\text {depol }}=25 \mathrm{~ns}$. The inset of Fig. 4a plots the Fourier transform of the experimental trace, depicting a strong

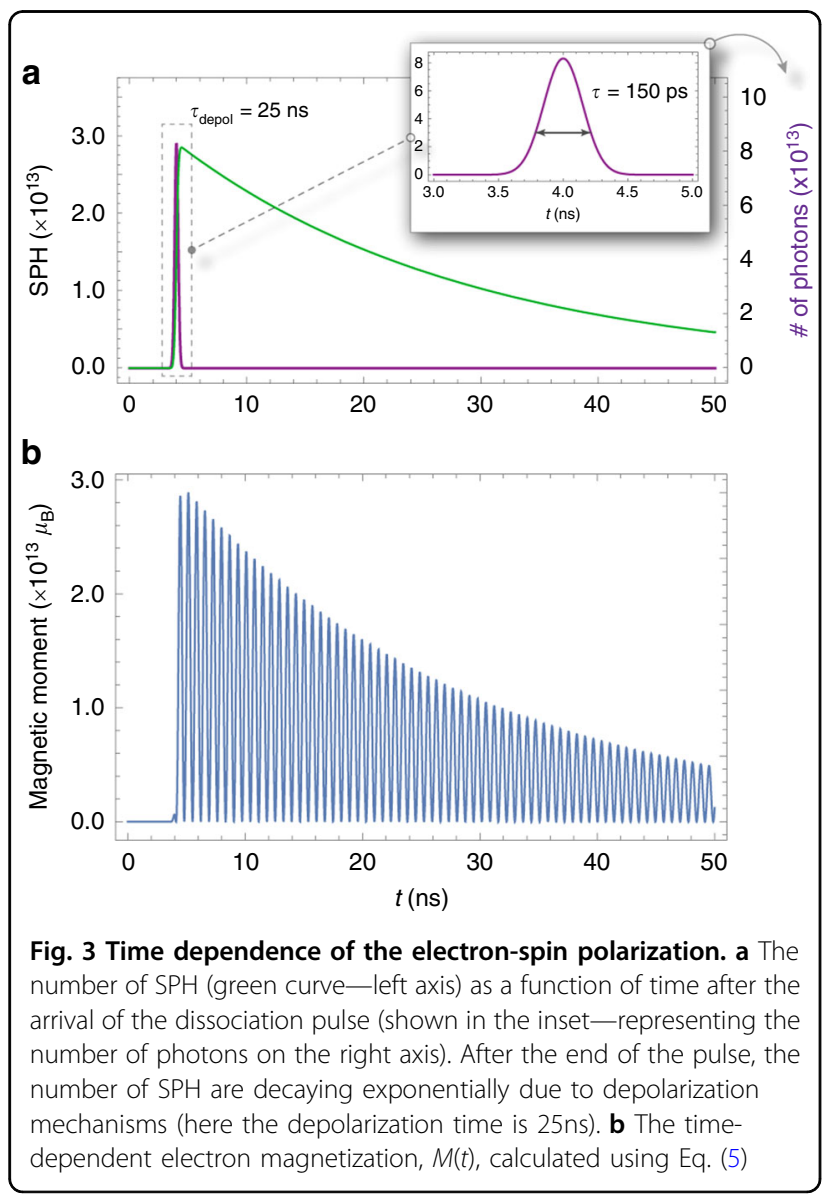

peak at $1.42 \mathrm{GHz}$, the $\mathrm{H}$ atom hyperfine frequency. The theoretical trace, calculated by solving Eq. (6) and using values of $\tau_{\text {depol }}=25 \mathrm{~ns}$ and $f=1.42 \mathrm{GHz}$, is shown in Fig. 4b.

Both signals resemble a damped oscillation; however, there are some more complicated features that are worth describing. First, both the theoretical and the experimental traces start with a rapid increase in $V(t)$, which relaxes soon (within two hyperfine periods). This rapid increase and its dynamics are related to the short laser pulse duration ( $150 \mathrm{ps}$ ) and are not related to the depolarization time $\tau$ at this pressure.

For negative $V(t)$, both the experimental and theoretical traces exhibit a smooth decrease before reaching the minimum value $V_{\min }(t) \approx-0.2 \mathrm{mV}$ and subsequently follow the depolarization exponential decay. This slower decrease in the negative $V(t)$ values is caused by the self-inductance of the detection coil. It is worth noting that by removing the second term in Eq. (3), this smooth decrease disappears from the theoretical trace, which becomes completely symmetric with respect to the $\mathrm{x}$ axis. We have seen, theoretically, that the effect of the detection-coil self-inductance on the signal gradually reduces for a lower frequency of the magnetization
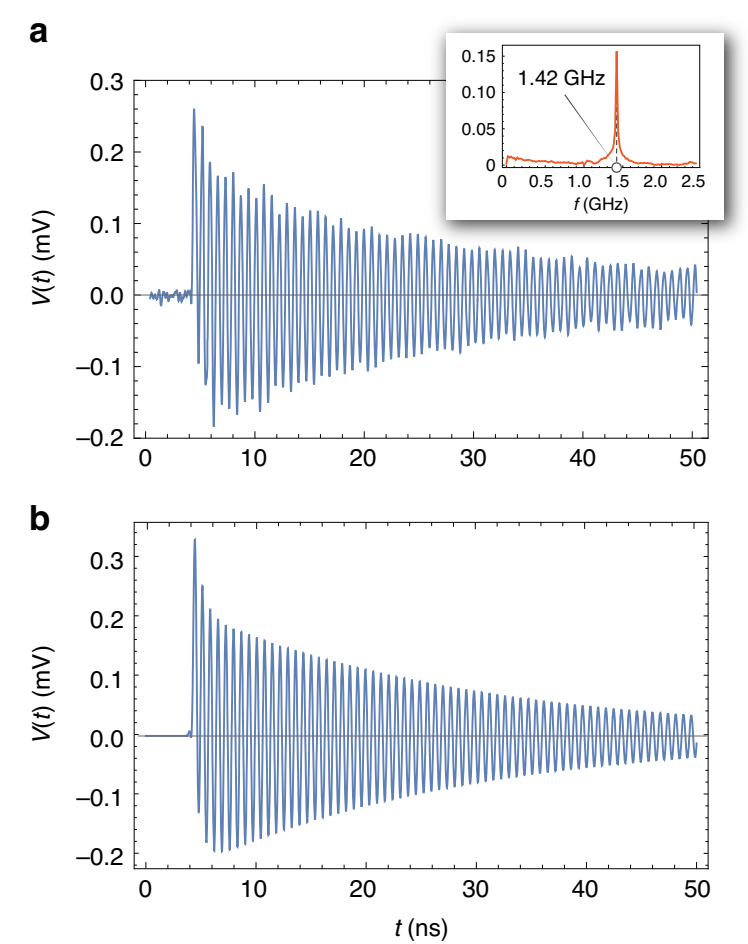

Fig. 4 SPH magnetization-quantum-beat signals. a Experimental SPH magnetization-quantum-beat signal from the photodissociation of $\mathrm{HBr}$ at $75 \mathrm{mbar}$, with a lifetime $\tau=25 \mathrm{~ns}$ and beating frequency $f=$ $1.42 \mathrm{GHz}$, shown by the Fourier transform (inset), and $\mathbf{b}$ Theoretical trace calculated using Eq. (6) (see text for details) 

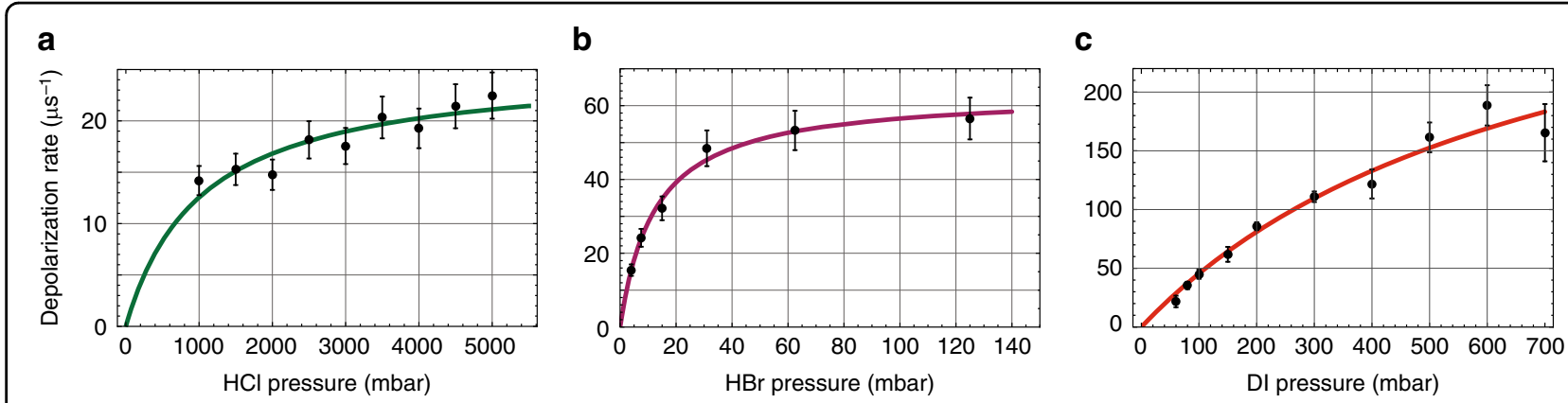

Fig. $5 \mathrm{SPH}$ depolarization rates. Measurements of the SPH depolarization rate as a function of hydrogen halide pressure ${ }^{35}$ for (a) $\mathrm{HCl}$, (b) $\mathrm{HBr}$, and (c) DI. The data are fit using Eq. (12)

oscillation and becomes negligible below a few tens of $\mathrm{MHz}$.

\section{SPH depolarization}

We produce SPH from the photodissociation of $\mathrm{HY}$ at high pressure, typically between $\mathrm{HY}$ densities of $10^{19}-10^{20} \mathrm{~cm}^{-3}(\sim 0.4-4$ bar HY pressure). If the photodissociation laser is unfocused, then only approximately $0.1 \%$ of the $\mathrm{HY}$ is photodissociated in the laser beam, and SPH is produced at densities of $\sim 10^{16} \mathrm{~cm}^{-3}$, surrounded by nonphotodissociated $\mathrm{HY}$ at high density. If the photodissociation laser is focused, then essentially all the HY can be photodissociated near the laser focus, and the SPH density approaches the initial HY density of $\sim 10^{19}-10^{20} \mathrm{~cm}^{-3}$. These two distinct density regimes, which we will call "low-density" and "high-density" SPH, show different depolarization mechanisms and will be discussed separately.

\section{Low-density SPH}

We note that "low-density" SPH production, of order $\sim 10^{16} \mathrm{~cm}^{-3}$, is still approximately four orders of magnitude higher than that of conventional SPH production methods. The two possible depolarizers are $\mathrm{Y}$ atoms (at the same "low density" as SPH) and nonphotodissociated $\mathrm{HY}$ at "high density", with bimolecular depolarization reactions:

$$
\begin{aligned}
& \mathrm{H}^{\uparrow}+\mathrm{Y} \stackrel{k^{Y}}{\rightarrow} \mathrm{H}+\mathrm{Y} \\
& \mathrm{H}^{\uparrow}+\mathrm{HY} \stackrel{k^{\mathrm{HY}}}{\rightarrow} \mathrm{H}+\mathrm{HY}
\end{aligned}
$$

with rate constants $k^{\mathrm{Y}}$ and $k^{\mathrm{HY}}$, respectively. These depolarization reactions predict linear depolarization as a function of $\mathrm{Y}$ and $\mathrm{HY}$ density ( $\mathrm{Y}$ density is proportional to HY density if the HY gas is not optically thick and limits the photodissociation light reaching the coil). However, the depolarization rates of $\mathrm{SPH} / \mathrm{SPD}$ as a function of $\mathrm{DI}, \mathrm{HBr}$, and $\mathrm{HCl}$ pressure are not linear but show curved behaviour that asymptotes to a plateau, as shown in Fig. 5. The explanation for this behaviour has been the proposal of depolarization through an intermediate complex, $\mathrm{HY}-\mathrm{H}^{\uparrow}$, where the $\mathrm{SPH}$ polarization is transferred to the $\mathrm{Y}$ nucleus through the hyperfine interaction and is subsequently transferred to $\mathrm{HY}-\mathrm{H}^{\uparrow}$ rotation and depolarized by collisions. The reaction steps are given by the three reactions ${ }^{35}$ :

$$
\begin{aligned}
& \mathrm{H}^{\uparrow}+\mathrm{HY} \underset{\mathrm{k}_{-1}}{\stackrel{\mathrm{k}_{1}}{\rightleftharpoons}} \mathrm{HY} \cdots \mathrm{H}^{\uparrow} \\
& \mathrm{HY} \cdots \mathrm{H}^{\uparrow} \stackrel{k_{d}}{\longrightarrow} \mathrm{HY} \cdots \mathrm{H} \\
& \mathrm{HY} \cdots \mathrm{H}^{\uparrow}+\mathrm{HY} \stackrel{k_{2}}{\longrightarrow} \mathrm{H}^{\uparrow}+2 \mathrm{HY}
\end{aligned}
$$

where Eq. (9) describes the complex formation and dissociation with rate constants $k_{1}$ and $k_{-1}$, respectively; Eq. (10) describes the nuclear hyperfine depolarization step, with rate constant $k_{\mathrm{d}}$, and Eq. (11) is the HY- $\mathrm{H}^{\uparrow}$ dissociation from collisions with HY, with rate constant $k_{2}$. Using the steady-state approximation and solving Eqs. (9-11) yields the $\mathrm{SPH}$ depolarization rate $K=1 / \tau$ :

$$
K=\frac{k_{1} k_{d}[H Y]}{\left(k_{-1}+k_{d}+k_{2}[H Y]\right)}
$$

Equation (12) is used for the excellent fits to the depolarization data in Fig. 5, and it predicts a plateau at a high [HY] of $\mathrm{K}_{\infty}=k_{1} k_{\mathrm{d}} / k_{2}$. Assuming $k_{1} / k_{2}$ is approximately constant for $\mathrm{HCl}, \mathrm{HBr}$, and DI, then $K_{\infty}=1 / \tau_{\infty}$ is proportional to $k_{\mathrm{d}}$, which is proportional to the hyperfine splitting of the complex and the quadrupole moment of the halogen nucleus. Indeed, it is observed that the highpressure SPH lifetimes $\tau_{\infty}=40 \mathrm{~ns}$ for $\mathrm{HCl}, 16 \mathrm{~ns}$ for $\mathrm{HBr}$, and $2.7 \mathrm{~ns}$ for DI scale approximately inversely proportional to the quadrupole moments of $\mathrm{Cl}, \mathrm{Br}$, and $\mathrm{I}$, respectively ${ }^{35}$. These lifetimes of 3-40 ns are sufficient for the applications described in sections V-VII. In addition, it is predicted that much longer lifetimes are possible 
from the photodissociation of HF because the $\mathrm{F}$ nucleus $\left(I_{\mathrm{F}}=1 / 2\right)$ lacks a quadrupole moment, and HF has hyperfine splittings approximately 2 orders of magnitude smaller than those of $\mathrm{HCl}$.

\section{High-density SPH}

When the $213 \mathrm{~nm}$ photodissociation laser pulse of $\sim 3 \mathrm{~mJ}$ is focused into the coil with a $50 \mathrm{~mm}$ lens, essentially all the HY molecules can be photodissociated, yielding SPH and Y atoms at the original HY density. In this case, the main SPH depolarization mechanism is from the collisions of $\mathrm{SPH}$ with $Y$ atoms, as shown in Eq. (7). The depolarization cross section for SPH from halogen atoms is not in the literature. The SPH depolarization cross section from alkali atoms is approximately $\sigma_{\text {alkali }} \sim 2 \times 10^{-15} \mathrm{~cm}^{2} 37$. If we assume that the $\mathrm{SPH}$ depolarization cross section from halogens is also $\sigma_{\text {halogen }} \sim 2 \times 10^{-15} \mathrm{~cm}^{2}$, then the rate constant $k_{\mathrm{Y}}=\sigma_{\text {halogen }}$ $\mathrm{v}$, where $\mathrm{v} \sim 2500 \mathrm{~m} / \mathrm{s}$ is the relative velocity of the $\mathrm{SPH}$ and $\mathrm{Y}$ collisions, gives $k_{\mathrm{Y}} \sim 5 \times 10^{-10} \mathrm{~cm}^{3} \mathrm{~s}^{-1}$. Therefore, for a density $[\mathrm{Y}]=10^{19} \mathrm{~cm}^{-3}$, the depolarization rate $k_{\mathrm{Y}}[\mathrm{Y}]=$ $5 \times 10^{9} \mathrm{~s}^{-1}$, yielding an SPH lifetime of $\sim 0.2 \mathrm{~ns}$. Under these conditions for DI, SPH lifetimes of approximately $\sim 10 \mathrm{~ns}$ longer were observed, and no evidence was seen for SPH depolarization from $\mathrm{I}\left({ }^{2} \mathrm{P}_{3 / 2}\right)$. The authors concluded that the upper limit for the SPH depolarization cross section $\sigma_{\text {halogen }} \leq 10^{-16} \mathrm{~cm}^{2}$ (at least an order of magnitude smaller than $\left.\sigma_{\text {alkali }}\right)$.

Another issue to consider is halogen atom recombination, which reduces SPH depolarization from halogen atoms, as well as the reaction of SPH with recombination product $\mathrm{Y}_{2}$, which removes $\mathrm{SPH}$. The recombination and $\mathrm{SPH}$ reactions are

$$
\begin{aligned}
& 2 \mathrm{Y}+\mathrm{Y} \stackrel{k_{r_{1}}}{\rightarrow} \mathrm{Y}_{2}+\mathrm{Y} \\
& 2 \mathrm{Y}+\mathrm{Y}_{2} \stackrel{k_{r_{2}}}{\rightarrow} 2 \mathrm{Y}_{2} \\
& \mathrm{H}^{\uparrow}+\mathrm{Y}_{2} \stackrel{k^{\mathrm{Y}_{2}}}{\rightarrow} \mathrm{HY}+\mathrm{Y}
\end{aligned}
$$

where $k_{r_{2}}=3.1(3) \times 10^{-30} \mathrm{~cm}^{6}$ molecule $\mathrm{e}^{-2} \mathrm{~s}^{-1}$ for $\mathrm{Y}=\mathrm{I}^{38}$, and $k_{r_{1}}=3.5 \times 10^{-33} \mathrm{e}^{810 / \mathrm{T}} \mathrm{cm}^{6}$ molecule $\mathrm{e}^{-2} \mathrm{~s}^{-1}$ for $\mathrm{Y}=$ $\mathrm{Cl}^{39}$ (where $\mathrm{T}$ is the gas temperature); $k_{r_{1}}$ is generally smaller than $k_{r_{2}}$ (as $\mathrm{Y}$ is smaller and has fewer internal degrees of freedom than $\mathrm{Y}_{2}$ ), for example, $k_{r_{1}}=8.75 \times$ $10^{-34} \mathrm{e}^{810 / \mathrm{T}} \mathrm{cm}^{6}$ for $\mathrm{Y}=\mathrm{Cl}^{39} ;$ for $\mathrm{Y}=\mathrm{Cl}, \quad k^{\mathrm{Y}_{2}}=$ $1.83 \times 10^{-11} \mathrm{~cm}^{3}$ molecule ${ }^{-1} \mathrm{~s}^{-1}$ at $298 \mathrm{~K}^{40}$, and $k^{\mathrm{Y}_{2}}=$ $6.3 \times 10^{-10} \mathrm{~cm}^{3}$ molecule ${ }^{-1} \mathrm{~s}^{-1}$ at $730 \mathrm{~K}^{41}$.

We note that for Eq. (15), the reaction cross section of $\mathrm{SPH}$ with $\mathrm{Cl}_{2}$ ranges from $\sim 10^{-16}$ to $\sim 3 \times 10^{-15} \mathrm{~cm}^{2}$ and is thus generally larger than the $\mathrm{SPH}-\mathrm{Cl}$ depolarization cross section. This means that any reduction in $\mathrm{SPH}$ depolarization from reactions (13) and (14) is counterbalanced by reaction (15). Fortunately, there is no $Y_{2}$ at $t=0$, and recombination is slow enough to prevent significant $Y_{2}$ formation at short times for all but the highest pressures. For example, for $[\mathrm{Cl}]=10^{20} \mathrm{~cm}^{-3}$ at $T=$ $298 \mathrm{~K}$, the initial recombination rate $k_{r_{1}}[\mathrm{Cl}]^{2} \sim 10^{8} \mathrm{~s}^{-1}$, showing that significant recombination occurs on timescales of approximately $10 \mathrm{~ns}$ and longer (similar times are found for $[\mathrm{I}]=10^{19} \mathrm{~cm}^{-3}$ ).

Immediately after photodissociation, both the $\mathrm{H}$ and $\mathrm{Y}$ atoms are polarized, as shown by Eq. (1). However, in the Fourier transform trace of the polarization beating signal (e.g., in Fig. 4a), only hyperfine beatings from SPH are observable, and no signals are seen from the halogen atoms (for $\mathrm{Cl}, \mathrm{Br}$, or I). This indicates that the halogen atoms are depolarized very rapidly (for example, on the $\sim 1 \mathrm{~ns}$ scale or faster). The depolarization cross sections of $\mathrm{Rb} 5 \mathrm{p}\left({ }^{2} \mathrm{P}_{3 / 2}\right)$ and $\mathrm{Cs} 6 \mathrm{p}\left({ }^{2} \mathrm{P}_{3 / 2}\right)$ states from collisions with noble gas atoms have been shown to be on the order of $10^{-14} \mathrm{~cm}^{242,43}$. If halogen atom $\left({ }^{2} \mathrm{P}_{3 / 2}\right)$-state depolarization is also on the order of $10^{-14} \mathrm{~cm}^{2}$, then we expect rapid depolarization of the halogen atoms on the $\sim 40$ ps timescale.

Therefore, the SPH lifetimes at "high density" of $\sim 10^{19}$ $\mathrm{cm}^{-3}$ and higher, in the presence of halogen atoms initially at similar density, are at least an order of magnitude longer than expected. The most plausible explanation is that the SPH halogen depolarization cross section is smaller than expected. Work is in progress to elucidate the SPH depolarization mechanism at "high density", and recent work indicates an upper limit for the depolarization cross section $\sigma_{\text {halogen }} \sim 10^{-16} \mathrm{~cm}^{29,44}$.

\section{Fast magnetometry}

Conventional atomic magnetometers typically can operate down to ms timescales ${ }^{45}$, whereas magnetometers based on nitrogen-vacancy centres in diamond have recently been shown to operate down to $\mu$ s timescales ${ }^{46}$. However, there are some applications that require ns or ps time resolution, such as the measurement of surface magnetism ${ }^{47}$, the generation of radical ion pairs in biochemical reactions such as photosynthesis ${ }^{48}$, and the ultrafast generation of magnetic fields with short-pulse lasers $^{49}$, with applications in laser fusion, particle-beam generation, and laboratory astrophysics.

The SPH production and detection method can be used for fast magnetometry, as the magnetization beating frequency is sensitive to the magnetic field, and it can be measured on the ns timescale. The time-dependent magnetic field can be determined from analysis of the beating trace in the time domain. In addition, the average magnetic field over the signal decay time (from approximately $10-100 \mathrm{~ns}$ ) can be determined by the Fourier transform of the signal. An example is shown in Fig. 6, where the hyperfine-beating peak at $1.42 \mathrm{GHz}$ (for $\mathrm{B}=0$ ) is split into two peaks by a field of $10.76 \mathrm{G}$. Detailed 
demonstrations of such fast magnetometry measurements are given elsewhere ${ }^{50}$, including demonstrations of nanosecond-resolved measurements with sensitivities of $\sim 10 \mathrm{mG} \mathrm{Hz}{ }^{-1 / 2}$ and proposals for achieving nanosecondresolved measurements with sensitivities down to at least the $\mu \mathrm{G}$ scale.

\section{Laser-plasma acceleration}

Polarized particle beams are used in scattering experiments in particle, nuclear, and solid-state physics. Conventional particle accelerators are typically very large and expensive facilities (often the size of $\sim 1 \mathrm{~km}$, or larger, e.g., the LHC at CERN). In recent years, laser-plasma acceleration has allowed the production of $\mathrm{MeV}$ and $\mathrm{GeV}$ highflux particle beams, accelerated over a distance of a few $\mathrm{cm}$. However, these high-flux beams are produced from the laser acceleration of an unpolarized gas jet, yielding a non-spin-polarized beam.

Our group proposed that our high-density SPH source can be used for laser acceleration of polarized particles ${ }^{9}$, as it is the only source that provides spin-polarized electrons, protons, or deuterons at the densities needed for laser-plasma acceleration. Subsequently, Wen et al. ${ }^{10}$ and then Wu et al. ${ }^{51}$ proposed that, using $\mathrm{SPH}$ from $\mathrm{HY}$ photodissociation, spin-polarized electron beams can be produced from laser acceleration, with fluxes of order $\sim 1 \mathrm{kA}$, or approximately 4 orders of magnitude larger than those from conventional sources ${ }^{10}$. Furthermore, they presented calculations that showed that the polarization of the electrons would be reduced by less than $10 \%$ due to the laser acceleration process. A similar proposal

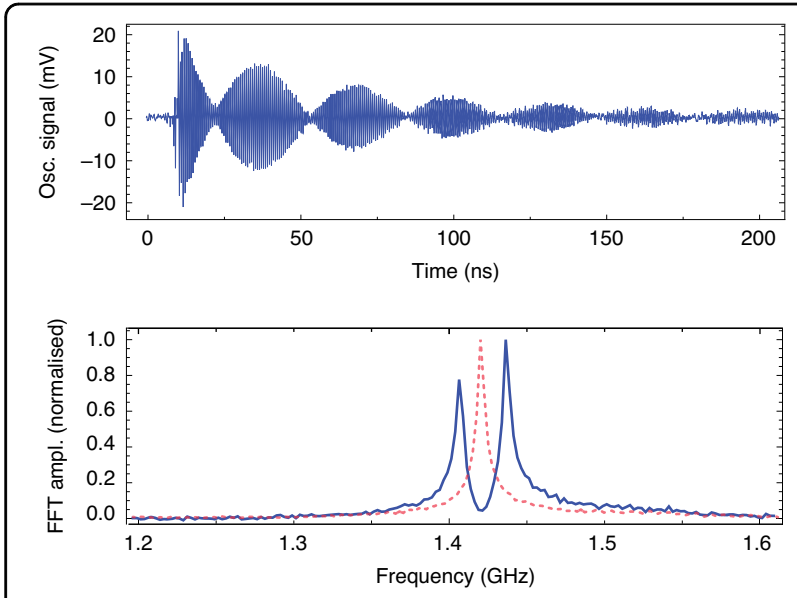

Fig. 6 The Zeeman effect. (Top) Experimental SPH magnetization beating trace, with a transverse magnetic field of $10.76 \pm 0.01 \mathrm{G}$. Notice the slow beating with a period of $\sim 30 \mathrm{~ns}$, caused by the beating of two frequencies. (Bottom) FFT of the top trace, showing that the trace (red dashed) without a magnetic field is split into two peaks (blue solid), due to the Zeeman effect. Clearly, this splitting, and the magnetic field strength, can be determined on the ns timescale was given by Hützen et al. ${ }^{52}$ for a laser-accelerated proton source, which also included experimental details needed to align the HY bonds with an IR laser to maximize the electron and nuclear polarization. Jin et al..$^{53}$ theoretically showed that proton polarization will be largely maintained during laser acceleration. A schematic of the experimental setup is given in Fig. 7. Briefly, $\mathrm{HY}$ gas is expanded supersonically through a nozzle into a vacuum chamber, so that just below the nozzle, the desired HY density, in the $10^{19}-10^{20} \mathrm{~cm}^{-3}$ range, is achieved. A focused infrared laser pulse aligns the HY bonds parallel to the axis of the linearly polarized IR light. At the peak of the IR pulse, when the HY molecules are fully aligned, a shorter, circularly polarized UV pulse photodissociates essentially all the HY molecules to produce SPH and halogen atoms. The halogen atoms can be removed by ionizing them with REMPI (for example, $\mathrm{Cl}\left({ }^{2} \mathrm{P}_{3 / 2}\right)$ atoms ionize at $234.62 \mathrm{~nm}$ via $2+1 \mathrm{REMPI}$ ); the electrons leave first, and the $\mathrm{Cl}^{+}$ions then leave the interaction region due to Coulomb explosion from the extremely high density of charge. The remaining high-density SPH atoms are used for laser-ion acceleration of spin-polarized electrons. In a similar setup, spin-polarized protons and deuterons can be accelerated; the removal of halogen atoms via ionization might not be necessary in this case because laser-accelerated protons or deuterons are separated from much heavier halogen nuclei. A review of the production of polarized beams from laser-plasma acceleration is given elsewhere ${ }^{54}$.

\section{Tests of polarized fusion}

The development of nuclear fusion as an energy source has been pursued for many years to address the increasing demand for clean energy with a small environmental impact. The two main methods, magnetic confinement

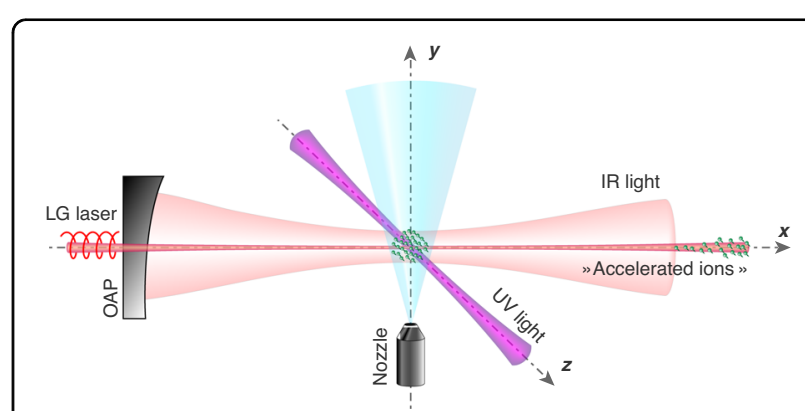

Fig. 7 Experimental setup for laser acceleration of highly spinpolarized protons, deuterons ${ }^{52,53}$, or electrons ${ }^{10,51}$. The nozzle produces an HY-molecule expansion in a vacuum chamber. An IR laser pulse aligns the HY bonds, and the circularly polarized UV laser photodissociates the HY molecules to produce SPH. The $\mathrm{Y}$ atoms can be removed by selective ionization with a UV laser. Finally, the spinpolarized protons, deuterons, or electrons from the SPH are ready for laser-driven wakefield acceleration by the high-power LG laser beam 
fusion (MCF) and inertial confinement fusion (ICF), are slowly approaching the break-even point, which needs to be exceeded for fusion to generate energy. Therefore, any contribution that can improve the efficiency of nuclear fusion can improve its viability as a commercial source of clean energy.

The three most important hydrogen isotope-based fusion reactions are deuterium-tritium (D-T), deuterium-helium-3 (D- $\left.{ }^{3} \mathrm{He}\right)$, and D-D:

$$
\begin{aligned}
& \mathrm{D}+\mathrm{T} \rightarrow{ }^{4} \mathrm{He}+\mathrm{n} \\
& \mathrm{D}+{ }^{3} \mathrm{He} \rightarrow{ }^{4} \mathrm{He}+\mathrm{p} \\
& \mathrm{D}+\mathrm{D} \rightarrow{ }^{3} \mathrm{He}+\mathrm{n} \\
& \mathrm{D}+\mathrm{D} \rightarrow \mathrm{T}+\mathrm{p}
\end{aligned}
$$

where $\mathrm{D}$ has a nuclear spin $\mathrm{I}_{\mathrm{D}}=1$, and $\mathrm{T}$ and ${ }^{3} \mathrm{He}$ have nuclear spins $\mathrm{I}_{\mathrm{T}}=\mathrm{I}_{\mathrm{He}-3}=1 / 2$.

It has been known for several decades that polarizing the nuclear spins in the $\mathrm{D}-\mathrm{T}$ and $\mathrm{D}-{ }^{3} \mathrm{He}$ reactions, Eqs. (16) and (17), increases the fusion cross sections by $50 \%$ (verified from scattering experiments) and can also increase the reactor fusion yield by approximately $75 \%{ }^{55,56}$. In contrast, it is not known what effect nuclear polarization has on the D-D reaction, as various difficult nuclear calculations are not in agreement, and no experiments have been performed ${ }^{57}$. In addition, it has not yet been demonstrated that nuclear polarization will survive long enough in the plasma to benefit nuclear fusion, although calculations indicate that it will for $\mathrm{ICF}^{58}$. In all these cases, the lack of tests of polarized fusion in a plasma stems from the inability of conventional methods to produce sufficient quantities of polarized $\mathrm{D}$ and $\mathrm{T}$, as well as the difficulty in transporting and introducing the polarized fuel into a reactor.

An intriguing possibility is offered by the production of high-density SPD and SPT from hydrogen halide photodissociation, for a relatively straightforward way to perform a test of polarized fusion. SPD can be produced at high density in situ, along with spin-polarized ${ }^{3} \mathrm{He}$ (or possibly SPT from TY photodissociation), and fusion events can be initiated with high-power lasers at laserfusion facilities. The angular distribution $D(\theta, \phi)$ of the neutron or proton products from the $\mathrm{D}-\mathrm{T}$ or $\mathrm{D}-{ }^{3} \mathrm{He}$ reactions is well-approximated by ${ }^{9,59}$

$$
D(\theta, \phi) \approx \frac{\sigma_{0}}{4 \pi}\left[(2+p)-\left(2 p+p_{z z}\right) P_{2}(\cos \theta)\right] / 3
$$

where $p=p_{z}(\mathrm{D}) p_{z}(\mathrm{~W}), \mathrm{W}$ is $\mathrm{T}$ or ${ }^{3} \mathrm{He}, p_{z}$ is the nuclear vector polarization, $p_{z z}$ is the tensor polarization for $\mathrm{D}$ nuclei (here $p_{z z}=0$ ), $\sigma_{0}$ is the unpolarized fusion cross section, and $P_{2}(\cos \theta)$ is the second Legendre polynomial.
Note that for $p=0, \mathrm{D}(\theta, \phi)$ is isotropic (independent of $\theta$ and $\phi)$. For the $\mathrm{D}_{-}{ }^{3} \mathrm{He}$ reaction performed with $p_{z}\left({ }^{3} \mathrm{He}\right)=$ $0.8^{60,61}$ and $p_{z}(\mathrm{D})= \pm 0.12^{9}$ (the + sign is selected for $\sigma^{+}$ circular photolysis light, and the - sign for $\sigma^{-}$light), giving $\mathrm{p}= \pm 0.1$, and the angular distribution of the products is given by $\mathrm{D}(\theta, \phi) \sim 1 \mp(0.1) \mathrm{P}_{2}(\cos \theta)$; this has approximately a $\pm 15 \%$ signal difference between product recoil directions of $\theta=0^{\circ}$ and $90^{\circ}$, and a $\pm 5 \%$ difference in the integrated intensity. By employing DI bond alignment and increasing the $\mathrm{D}$ polarization by approximately a factor of 4 (giving $p_{z}(\mathrm{D})= \pm 0.5$ and $p= \pm 0.4$ ), the signal effects are also increased by a similar factor. Similar experiments can likely be performed for $\mathrm{D}-\mathrm{T}$, replacing polarized ${ }^{3} \mathrm{He}$ with polarized $\mathrm{T}$ from tritium halide photodissociation because the $\mathrm{D}-\mathrm{T}$ fusion cross section is approximately 100 times larger than that for $\mathrm{D}^{3} \mathrm{He}$, yielding a much higher product yield. However, neither theory nor experiments have yet been performed on tritium halide photodissociation to verify that highly polarized $\mathrm{T}$ is produced.

The appeal of such tests of laser fusion is the simplicity of execution and that no further experimental development is needed. If it is shown that nuclear polarization survives ICF plasma and benefits fusion, then this may motivate the complicated development of nuclear spinpolarized fusion pellets, which are currently beyond the capabilities of conventional methods.

\section{Conclusions}

SPH can be produced via the photodissociation of hydrogen halide molecules at extremely high densities of at least $10^{19} \mathrm{~cm}^{-3}$ and on very short timescales (fs-ns) using pulsed lasers (these densities and timescales are approximately 7 orders of magnitude higher and 10 orders of magnitude faster than conventional methods, respectively). The SPH depolarization times are surprisingly long, especially at high densities, as the depolarization cross section of SPH by halogen atoms seems to be smaller than expected. This new regime of high-density $\mathrm{SPH}$ opens up new applications for SPH, including laserion acceleration of polarized particles, ultrafast magnetometry, and tests of polarized nuclear fusion.

\section{Acknowledgements}

This work is supported by the Hellenic Foundation for Research and Innovation (HFRI) and the General Secretariat for Research and Technology (GSRT), grant agreement No. HFRI-FM17-3709 (project NUPOL) and by the project "HELLAS$\mathrm{CH}^{\prime}$ (MIS 5002735), which is implemented under the "Action for Strengthening Research and Innovation Infrastructures", funded by the Operational Programme "Competitiveness, Entrepreneurship and Innovation" (NSRF 20142020) and cofinanced by Greece and the European Union (European Regional Development Fund). GK acknowledges funding from the Hellenic Foundation for Research and Innovation (HFRI) and the General Secretariat for Research and Technology (GSRT), under project HANDCORE grant agreement No 1789 Figure 5 is adapted with permission from Boulogiannis et al., "Spin-polarized hydrogen depolarization rates at high hydrogen halide pressures: hyperfine depolarization via the HY-H complex", The Journal of Physical Chemistry A $\mathbf{1 2 3}$, 
8130-8134 (2019). Copyright (2019) American Chemical Society. Figure 7 is adapted with permission from Wu et al., "Polarized electron-beam acceleration driven by vortex laser pulses" New Journal of Physics 21, 073052 (2019), (https:// creativecommons.org/licenses/by/3.0/).

\section{Author details}

${ }^{1}$ Foundation for Research and Technology Hellas, Institute of Electronic Structure and Laser, N. Plastira 100, Heraklion, Crete GR-71110, Greece. ${ }^{2}$ University of Crete, Department of Physics, Herakleio, Greece. ${ }^{3}$ Department of Physics, Atomic and Molecular Physics Laboratory, University of Ioannina, University Campus, Ioannina GR-45110, Greece

\section{Conflict of interest}

The authors declare that they have no conflict of interest.

Supplementary information The online version contains supplementary material available at https://doi.org/10.1038/s41377-021-00476-y.

Received: 30 May 2020 Revised: 8 January 2021 Accepted: 18 January 2021 Published online: 12 February 2021

\section{References}

1. Steffens, E. \& Haeberli, W. Polarized gas targets. Rep. Prog. Phys. 66, 1887-1935 (2003).

2. Leader, E. Spin in Particle Physics (Cambridge University Press, 2001).

3. Paetz gen. Schieck, H. The status of "polarized fusion". Eur. Phys. J. A 44, 321-354 (2010)

4. Delgado, F. \& Fernández-Rossier, J. Spin decoherence of magnetic atoms on surfaces. Prog. Surf. Sci. 92, 40-82 (2017).

5. Leggett, A. J. Bose-Einstein condensation in the alkali gases: some fundamental concepts. Rev. Mod. Phys. 73, 307-356 (2001).

6. Budker, D. \& Romalis, M. Optical magnetometry. Nat. Phys. 3, 227-234 (2007)

7. Ardenkjaer-Larsen, J. H. et al. Facing and overcoming sensitivity challenges in biomolecular NMR spectroscopy. Angew. Chem. Int. Ed. 54, 9162-9185 (2015).

8. Oros, A. M. \& Shah, N. J. Hyperpolarized xenon in NMR and MRI. Phys. Med. Biol. 49, R105-R153 (2004).

9. Sofikitis, D. et al. Ultrahigh-density spin-polarized $H$ and $D$ observed via magnetization quantum beats. Phys. Rev. Lett. 121, 083001 (2018).

10. Wen, M., Tamburini, M. \& Keitel, C. H. Polarized laser-WakeField-accelerated Kiloampere electron beams. Phys. Rev. Lett. 122, 214801 (2019).

11. Szczerba, D. et al. A polarized hydrogen/deuterium atomic beam source for internal target experiments. Nucl. Instrum. Methods Phys. Res. A: Accelerators, Spectrometers, Detect. Associated Equip. 455, 769-781 (2000).

12. Clasie, B. et al. Laser-driven target of high-density nuclear-polarized hydrogen gas. Phys. Rev. A 73, 020703 (2006)

13. Redsun, S. G. et al. Production of highly spin-polarized atomic hydrogen and deuterium by spin-exchange optical pumping. Phys. Rev. A 42, 1293-1301 (1990).

14. Eppink, A. T. J. B. et al. Production of maximally aligned $O\left({ }^{1} D\right)$ atoms from twostep photodissociation of molecular oxygen. J. Chem. Phys. 108, 1305-1308 (1998).

15. Rakitzis, T. P. \& Kitsopoulos, T. N. Measurement of Cl and Br photofragment alignment using slice imaging. J. Chem. Phys. 116, 9228-9231 (2002).

16. Chestakov, D. A. et al. Photofragment alignment in the photodissociation of $\mathrm{I}_{2}$ from 450 to $510 \mathrm{~nm}$. J. Chem. Phys. 124, 024315 (2006).

17. Van Brunt, R. J. \& Zare, R. N. Polarization of atomic fluorescence excited by molecular dissociation. J. Chem. Phys. 48, 4304-4308 (1968).

18. Vasyutinskiil, O. S. Orientation of atoms during photodissociation of molecules. JETP Lett. 31, 428-430 (1980).

19. Rakitzis, T. P. et al. Spin-polarized hydrogen atoms from molecular Photodissociation. Science 300, 1936-1938 (2003).

20. Rakitzis, T. P. Pulsed-laser production and detection of spin-polarized hydrogen atoms. ChemPhysChem 5, 1489-1494 (2004).

21. Rakitzis, T. P. et al. Measurement of $\mathrm{Br}$ photofragment orientation and alignment from $\mathrm{HBr}$ photodissociation: production of highly spin-polarized hydrogen atoms. J. Chem. Phys. 121, 7222-7227 (2004).

22. Rubio-Lago, L. et al. Laser preparation of spin-polarized atoms from molecular photodissociation. Phys. Scr. 73, C71-C75 (2006).
23. Sofikitis, D. et al. Nanosecond control and high-density production of spinpolarized hydrogen atoms. EPL (Europhys. Lett.) 81, 68002 (2008).

24. Sofikitis, D. et al. Laser detection of spin-polarized hydrogen from $\mathrm{HCl}$ and $\mathrm{HBr}$ photodissociation: Comparison of $\mathrm{H}$ - and halogen-atom polarizations. J. Chem. Phys. 129, 144302 (2008)

25. Bougas, L. et al. $(2+1)$ laser-induced fluorescence of spin-polarized hydrogen atoms. J. Chem. Phys. 133, 174308 (2010).

26. Broderick, B. M. et al. Velocity distribution of hydrogen atom spin polarization. J. Phys. Chem. Lett. 4, 3489-3493 (2013).

27. Broderick, B. M. et al. Spin-polarized hydrogen Rydberg time-of-flight: experimental measurement of the velocity-dependent $H$ atom spinpolarization. Rev. Sci. Instrum. 85, 053103 (2014).

28. Broderick, B. M. et al. Imaging detection of spin-polarized hydrogen atoms. Chem. Phys. Lett. 635, 350-354 (2015).

29. Sofikitis, D. et al. Highly nuclear-spin-polarized deuterium atoms from the UV photodissociation of deuterium lodide. Phys. Rev. Lett. 118, 233401 (2017).

30. Balint-Kurti, G. G. et al. Vector correlations and alignment parameters in the photodissociation of HF and DF. J. Chem. Phys. 116, 10760-10771 (2002).

31. Brown, A., Balint-Kurti, G. G. \& Vasyutinskii, O. S. Photodissociation of HCL and DCL: polarization of atomic photofragments. J. Phys. Chem. A 108, 7790-7800 (2004).

32. Brown, A. Photodissociation of $\mathrm{HI}$ and $\mathrm{Dl}$ : polarization of atomic photofragments. J. Chem. Phys. 122, 084301 (2005)

33. Jodoin, D. N. \& Brown, A. Photodissociation of HI and Dl: testing models for electronic structure via polarization of atomic photofragments. J. Chem. Phys. 123, 054301 (2005)

34. Sofikitis, D. et al. Photofragment spin-polarization measurements via magnetization quantum beats: dynamics of DI photodissociation. Phys. Chem. Chem. Phys. 21, 14000-14004 (2019).

35. Boulogiannis, G. K. et al. Spin-polarized hydrogen depolarization rates at high hydrogen halide pressures: hyperfine depolarization via the HY-H complex. J. Phys. Chem. A 123, 8130-8134 (2019).

36. Milner, A. A., Korobenko, A. \& Milner, V. Ultrafast magnetization of a dense molecular gas with an optical centrifuge. Phys. Rev. Lett. 118, 243201 (2017).

37. Zelenski, A. N. \& Kokhanovski, S. A. A study of spin-exchange polarization transfer in hydrogen-rubidium collisions. AIP Conf. Proc. 293, 164-172 (1993).

38. Ip, J. K. K. \& Burns, G. Recombination of iodine atoms by flash photolysis over a wide temperature range. $\| \mathrm{I}_{2}$ in $\mathrm{He}, \mathrm{Ar}, \mathrm{Xe}, \mathrm{N}_{2}, \mathrm{CO}$. J. Chem. Phys. 56, 3155-3161 (1972)

39. Kemaneci, E. et al. Global (volume-averaged) model of inductively coupled chlorine plasma: influence of $\mathrm{Cl}$ wall recombination and external heating on continuous and pulse-modulated plasmas. Plasma Sources Sci. Technol. 23 045002 (2014).

40. Bykhalo, I. B. et al. Kinetics study of two-channel hydrogen and deuterium atom reactions with interhalogen molecules. Russian Chem. Bull. 43, 1637-1645 (1994).

41. Baulch, D. L. et al. Evaluated kinetic data for high temperature reactions Volume 4: homogeneous gas phase reactions of halogen- and cyanidecontaining species. J. Phys. Chem. Ref. Data 1, 10 (1981).

42. Gallagher, A. Collisional depolarization of the Rb $5 p$ and Cs $6 p$ doublets. Phys. Rev. 157, 68-72 (1967).

43. Bayram, S. B. et al. Collisional depolarization of Zeeman coherences in the ${ }^{133} \mathrm{Cs} 6 p^{2} P_{3 / 2}$ level: double-resonance two-photon polarization spectroscopy. Phys. Rev. A 73, 042713 (2006).

44. Spiliotis, A. K et al. Depolarization of spin-polarized hydrogen via spinexchange collisions with chlorine atoms at ultrahigh density. Preprint at https://arxiv.org/abs/2101.02675 (2021).

45. Jiménez-Martínez, R. et al. High-bandwidth optical magnetometer. J. Optical Soc. Am. B 29, 3398-3403 (2012).

46. Zheng, $\mathrm{H}$. J. et al. Microwave-free vector magnetometry with nitrogenvacancy centers along a single axis in diamond. Phys. Rev. Appl. 13, 044023 (2020).

47. Sirotti, F. et al. Time-resolved surface magnetometry in the nanosecond scale using synchrotron radiation. J. Appl. Phys. 83, 1563-1568 (1998).

48. Link, G. et al. High time resolution multifrequency EPR of radical pair intermediates in photosynthetic reaction centers: Structure determination on a nanosecond time scale. Z. Physik. Chem. 218, 171-191 (2004).

49. Zhu, B. J. et al. Ultrafast pulsed magnetic fields generated by a femtosecond laser. Appl. Phys. Lett. 113, 072405 (2018).

50. Spiliotis, A. K. et al. A nanosecond-resolved atomic hydrogen magnetometer. Preprint at https://arxiv.org/abs/2010.14579 (2020). 
51. Wu, Y. T. et al. Polarized electron-beam acceleration driven by vortex laser pulses. N. J. Phys. 21, 073052 (2019).

52. Hützen, $\mathrm{A}$. et al. Polarized proton beams from laser-induced plasmas. High Power Laser Sci. Eng. 7, e16 (2019).

53. Jin, L. L. et al. Spin-polarized proton beam generation from gas-jet targets by intense laser pulses. Phys. Rev. E 102, 011201 (2020).

54. Büscher, M. et al. Polarized beams from laser-plasma accelerators. High. Power Laser Sci. Eng. 8, e36 (2020).

55. Temporal, M. et al. Ramis. Ignition conditions for inertial confinement fusion targets with a nuclear spin-polarized DT fuel. Nucl. Fusion 52, 103011 (2012).
56. Hupin, G., Quaglioni, S. \& Navrátil, P. Ab initio predictions for polarized deuterium-tritium thermonuclear fusion. Nat. Commun. 10, 351 (2019).

57. Engels, R. et al. Polarized fusion. Phys. Part. Nucl. 45, 341-343 (2014).

58. More, R. M. Nuclear spin-polarized fuel in inertial fusion. Phys. Rev. Lett. $\mathbf{5 1}$ 396-399 (1983).

59. Kulsrud, R. M. et al. Fusion reactor plasmas with polarized nuclei. Phys. Rev. Lett. 49, 1248-1251 (1982).

60. Batz, M. et al. ${ }^{3} \mathrm{He}$ spin filter for neutrons. J. Res. Natl Inst. Stand. Technol. 110, 293-298 (2005).

61. Walker, T. G. \& Happer, W. Spin-exchange optical pumping of noble-gas nuclei. Rev. Mod. Phys. 69, 629-642 (1997). 